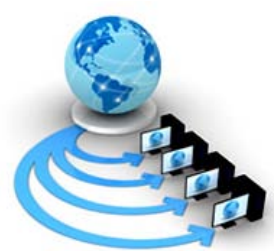

Volume 8, No. 7, July - August 2017

International Journal of Advanced Research in Computer Science

RESEARCH PAPER

Available Online at www.ijarcs.info

\title{
THE IMPACT OF CROWDSOURCING IN SOFTWARE ENGINEERING: SOFTWARE TESTING PERSPECTIVE
}

\author{
Anoop Upadhyay \\ Department of Information Technology \\ Babasaheb Bhimrao Ambedkar University \\ Lucknow, India
}

\author{
Mohd Waris Khan \\ Department of Information Technology \\ Babasaheb Bhimrao Ambedkar University \\ Lucknow, India
}

\author{
Pawan Kumar Chaurasia \\ Department of Information Technology \\ Babasaheb Bhimrao Ambedkar University \\ Lucknow, India
}

\begin{abstract}
In current scenario, a lot of crowdsourcing systems have come out and lead to numerous successful crowdsourcing systems like Defense Advanced Research Projects Agency (DARPA), pay4bugs, Wiki-pedia, Waze, bugfinders, Facebook, Amazon, etc. Security concern in the software application domain is without difficulty browbeaten by the attackers. In S/w engineering, crowdtesting has acquired increased interest and acceptance, particularly among developer and smaller organizations. In this paper, we stipulate a comprehensive survey of the use of crowdsourcing in software engineering and the importance of crowdsourcing in S/w engineering with respect to software testing is also elaborated. Further, we analyze the problems related to security point of view. This process maximizes the success of the software system. According to the recent trend, the croudtesting is gaining cognizance from the industries, experts and research communities. Hence implications of these flaws in the performance of software models' have been explored.
\end{abstract}

Keywords: Crowdsourcing, crowdtesting, Security Factors, etc.

\section{INTRODUCTION}

Crowdsourcing means the act of an organization or institution taking a function once performed by employees and outsourcing it to an undefined network of people in the form of an open call. Crowdsourced Testing subsist in entrusting software testing tasks to a community of connoisseur quality assurance testers through an online platform. It is quickly emerging as the most efficient way to streamline interactive software production and improve the quality of the web and software products published by developers [1, 3]. Notable representation of this novel model includes Amazon 4bugs, Wiki-pedia, Waze, bugfinders, Facebook and user-generated advertising contests. All of these systems are very successful which illustrates that crowdsoucing can take advantage of the cognitive content of crowd with a low cost and get great outputs.

As crowdsourcing has met extraordinary success in many fields, can we adopt this model into software engineering area? Actually, open source software has adopted crowdsourcing for software development for years. In the development process of open source software, software's design or blueprint is universally accessible via free license and anyone can provide improvements to it which helps make the software robust and stable [2]. Crowdtesting enables software developers to get their products tested on all major platforms, devices, system configurations and country or region-specic aspects under realworld conditions. With crowdtesting, the developers can get test results on functional testing, security testing, load \& performance testing, usability testing and localization testing with reduced costs in shorter time [4, 5].
The remaining part of this paper is organized as follows: Section 2 introduces the numerous advantages Crowdsourced testing methodology on literature search. Section 3 provides a finer-grained view of Crowdsourced Software Engineering and role of crowdsourced testing in software development lifecycle. Section 4 presents relation b/w crowdsourcing and software testing. Section 5 presents detailed about Crowdsourced Testing delivers with software testing services. Sections 6 defines the conclusion of the research paper.

\section{ADVANTAGES OF CROWDSOURCE TESTING}

Flexibility: Given that our crowd comprehends testers in all time zones, there are always testers accessible, no matter the time of day. This comes in peculiarly convenient when working late at night trying to meet a deadline [6].

Instantaneity: distinct traditional software testing organizations, Crowdsourced Testing services are accessible on short notice and allow you to get results in as little as a few hours.

Realworld multistage testing: When dealing with external client, products are likely to be used on a much wider variety of hardware and software combinations otherthan personal. These include older browser versions, other operating systems and different hardware devices. A large crowd of testers is working in real world so, it is important to ensure that the product is tested in various real world situations and on a large variety of hardware and software combinations. 


\section{CROWDSOURCED SOFTWARE ENGINEERING}

The term 'Crowdsourced Software Engineering' is use to denote the application of crowdsourcing techniques to support software development. However, we prefer the term 'Crowdsourced Software Engineering' since it emphasises any software engineering activity included, thereby encompassing performance that do not essentially yield software in themselves [15]. Example activities include project planning, requirements elicitation, security augmentation and test case refinement.

However, although our definition is inclusive of all software engineering activities, it is important to distinguish Crowdsourced Software Engineering from the research activities on software engineering that happen to be supported by Crowdsourcing (see Fig. 1 ) [9]. Any research concerning human subjects could potentially be sustained by crowdsourcing, in the sense that the identification and recruitment of suitable human subjects for an experiment could be executed using crowdsourcing techniques.

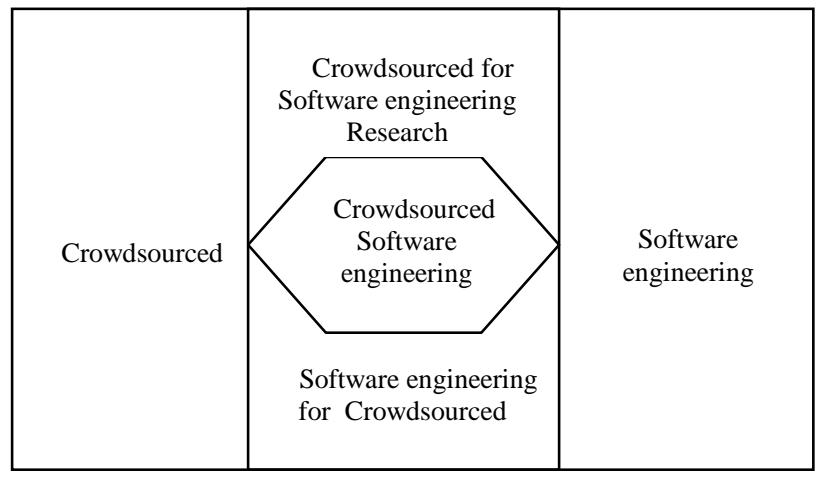

Fig.1 Crowdsourced and Software Engineering

In this application of crowdsourcing, it is the identification of human subjects for experimentation that is important, rather than the particular research topic explored. When the research topic happens to be software engineering, then this work will be fascinating to software engineers, but the ideology and problems that arise will be more similar and related to those occuring in other study concerning with human subjects. In this paper, we comprehensively survey Crowdsourced Software Engineering. We do not claim to cover crowdsourcing for software engineering research as comprehensively. In addition, Fig. 1 represents, software engineering techniques can also be used to support the implementation of generic crowdsourcing, e.g., building a novel crowdsourcing system to support the crowdsourcing process.

\section{RELATION BETWEEN CROWDSOURCING AND SOFTWARE TESTING}

Creative thinking is challenging to crowdsource or outsource. How a crowdsource design? It is possible, but it might be insight hard to find people who will convey your vision exactly as you would have appealing. It may be possible when the staff/employ is talented designer or developer, otherwise a lot trouble have been faced during testing [7, 9].

The complete web or S/w development process can be called original. From beginning to industry, products are constantly germinated and the staff/employ makes creative decisions until the very end $[8,10]$. However, the $S / w$ testing process represents a lead role of the creative interactive development process which is entirely logical. S/w testing is about consideration to facet and perfectionism, while quality assurance tests evaluate the quality of an interactive product and making suggestions on how it can be improved, the unlucky reality is that they are often conducted by rushed copywriters or project managers with very limited time at their administration.

As a project manager, it is very important to find out the solution of the problem when the situations were not in favour. When the suspecting file contained bugs are discover through testing and a lot of pressure is releases when bugs are detected otherwise so much pressure to deliver on the staff and the excuses are given that we didn't have too much of time to properly test it [11]. Programmers ran late, the team kept finding bugs, and we would finally ship the product several hours late without proper testing, hoping that our prayers would be heard. Crowdsourced testing recommended the advantage of facilitating companies to carry out tests when it is most suitable for them, no matter the time or day of the week [12]. In this way the programmers can create a test request before they leave work station and return to find a comprehensible and summarizing list of bugs to fix those problem. This may help to save an huge amount of time and money.

\section{How Crowdsourced Testing Delivers EXCELLENT SOFTWARE TESTING SERVICES}

At Crowdsourced Testing, it is necessary to continuously review the work of every tester participating in software testing projects to ensure great results $[3,13]$. Further, a tester gets concerned in projects and the enhanced his work, the more likely the tester present confidence to test our clients' projects. Communication with testers is very important for client so when properly communication takes place the client won't suffer through the pitfalls often associated with traditional crowdsourcing: poor results, incomplete bug descriptions, or language barriers.

The main task is to help web and software developers deliver their projects on time and on budget by providing them with excellent quality assurance testing services at flexible hours. when testers know about the clients for a long time and understand reality then testers easily understood the client problem and resolve those problem smoothly $[4,14]$. The result creates a great impact on crowdsourced software testing. Hence, the output witnessed increased interest and adoption, meanwhile, many crowdsourced testing products have arise.

\section{CONCLUSION}

In this paper, we have examine the existing literature on the use of crowdsourcing in software engineering activities and research into these activities. The study has revealed a progressively increasing rate of publication. Specifically, the crowdsource testing is defined and relation $\mathrm{b} / \mathrm{w}$ crowdsource and software tesing may improve the quality of the software applications. The study also highlights latent issues in Crowdsourced Software Engineering, together with related analysis and solutions conducted in previous studies. Finally, this paper is used to identify the importance and open problems for future work. 


\section{REFERENCES}

[1] K. Mao, L. Capra, M. Harman, Y. Jia, "A survey of the use of crowdsourcing in software engineering," Journal of Systems and Software, Elsevier, 2016.

[2] M W Khan, D. Pandey, S A Khan, "Critical Review on Software Testing: Security Perspective,” CCIS Series628, Springer, pp. 714-723, 2016.

[3] Minzhi Yan, Hailong Sun, Xudong Liu, "iTest: Testing Software with Mobile Crowdsourcing," School of Computer Science and Engineering, Beihang University, Beijing, China, 17 November 2014.

[4] R. Pham, L. Singer and Kurt Schneider. Building Test Suites in Social Coding Sites by Leveraging Drive-By Commits. 35th International Conference on Software Engineering, pp. 12091212, May, 2013.

[5] TopCoder. http://www.topcoder.com

[6] Klaas-Jan Stol Lero, “Two's Company, Three's a Crowd: A Case Study of Crowdsourcing Software Development”, The Irish Software Engineering Research Centre, University of Limerick, Ireland, 2014.

[7] M W Khan, V. Katiyar, D Pandey, N Tiwari and R. Kumar, "Role of Requirement Engineering in E-Learning Services," Global Journal of Multidisciplinary Studies, ISSN: - 2348-0459, Volume 3, Issue 5, April-2014.
[8] Matt Johnston Applause Framingham, "Software Crowdsourcing Practices and Research Directions," Symposium on Service-Oriented System Engineering, IEEE, USA, 2016.

[9] Ke Mao , Licia Capra , Mark Harman , Yue Jia, “A survey of the use of crowdsourcing in software engineering" The Journal of Systems and Software 126, Elsevier, pp.57-84, 2017.

[10] Wei-Tek Tsai, Wenjun Wu, and Michael N. Huhns. "Cloudbased software crowdsourcing," IEEE Internet Computing 3 pp.78-83, 2014.

[11] Wenjun Wu, Wei-Tek Tsai, and Wei Li. "An evaluation framework for software crowdsourcing," Frontiers of Computer Science 7.5, pp.694-709, 2013.

[12] Kazman, Rick, and Hong-Mei Chen. "The metropolis model a new logic for development of crowdsourced systems," Communications of the ACM 52.7 pp.76-84, 2013.

[13] Hu, Zhenghui, and Wenjun Wu. "A Game Theoretic Model of Software Crowdsourcing,", IEEE 8th International Symposium on Service Oriented System Engineering (SOSE), 2014.

[14] Hu, Zhenghui, and Wenjun Wu. "A Game Theoretic Model of Software Crowdsourcing,", IEEE 8th International Symposium on Service Oriented System Engineering (SOSE), 2014.

[15] Kazman, Rick, and Hong-Mei Chen. "The metropolis model a new logic for development of crowdsourced systems," Communications of the ACM 52.7, pp.76-84, 2013. 